\title{
Validation of the Numerical Rating Scale for Pain Intensity and Unpleasantness in Pediatric Acute Postoperative Pain: Sensitivity to Change Over Time
}

\author{
M. Gabrielle Pagé, ${ }^{*}$ Joel Katz, ${ }^{*},+\uparrow$ Jennifer Stinson, ${ }^{\ddagger \S}$ Lisa Isaac, ${ }^{\dagger, \ddagger}$ \\ Andrea L. Martin-Pichora, ${ }^{*}$ and Fiona Campbell ${ }^{\dagger, *}$ \\ *Department of Psychology, Faculty of Health, York University, Toronto, ON, Canada. \\ ${ }^{\dagger}$ Department of Anesthesia, Faculty of Medicine, and ${ }^{\S}$ Faculty of Nursing, University of Toronto, Toronto, ON, Canada. \\ Departments of ${ }^{\ddagger}$ Anesthesia and Pain Medicine, and ${ }^{\top}$ Psychology, Hospital for Sick Children, Toronto, ON, Canada.
}

\begin{abstract}
This study evaluates the construct validity (including sensitivity to change) of the numerical rating scale (NRS) for pain intensity (I) and unpleasantness (U) and participant pain scale preferences in children/adolescents with acute postoperative pain. Eighty-three children aged 8 to 18 years (mean $=13.8, S D=2.4$ ) completed 3 pain scales including NRS, Verbal Rating Scale (VRS), and faces scales (Faces Pain Scale-Revised [FPS-R] and Facial Affective Scale [FAS], respectively) for pain intensity (I) and unpleasantness (U) 48 to 72 hours after major surgery, and the NRS, VRS and Functional Disability Index (FDI) 2 weeks after surgery. As predicted, the NRSI correlated highly with the VRSI and FPS-R and the NRSU correlated highly with the VRSU and FAS $\mathbf{4 8}$ to 72 hours after surgery. The FDI correlated moderately with the NRS at both time points. Scores on the NRSI and NRSU at 48 to 72 hours were significantly higher than at 2 weeks after surgery. Children found the faces scales the easiest to use while the VRS was liked the least and was the hardest to use. The NRS has adequate evidence of construct validity including sensitivity for both pain intensity and unpleasantness. This study further supports the validity of the NRS as a tool to measure both intensity and unpleasantness of acute pain in children.

Perspective: This article evaluates the construct validity including sensitivity of the Numerical Rating Scale for pain intensity and pain unpleasantness over time in children after major surgery. The NRS could be used by clinicians to assess these 2 different dimensions of children's pain experience in acute pain settings.
\end{abstract}

$\mathrm{T}$ he numerical rating scale (NRS) is one of the simplest and most frequently used instruments in clinical practice to measure a child's pain intensity in children 8 years and older. ${ }^{34}$ Children verbally rate the intensity of pain on a scale from " 0 " (no pain) to " 10 " (worst pain possible; most hurt possible). In contrast to the abundance of data on the reliability and validity of other pediatric pain scales, such as the Oucher, ${ }^{3,4}$ Faces Pain Scale-Revised (FPS-R), ${ }^{17}$ Pieces of Hurt Scale, ${ }^{16}$ the Visual Analog Scale (VAS), ${ }^{24,33}$ and the Wong-Baker FACES Pain Rating Scale, ${ }^{40}$ very few studies have evaluated the psychometric properties of the NRS. $1,2,7,26,34,35,37$ The NRS is recommended for use in adult clinical trials (IMMPACT), ${ }^{10}$ but it was not selected for use in children due to a lack of psychometric evidence. ${ }^{25}$ Whereas the scales listed above are well supported empirically, ${ }^{34}$ unlike the NRS, they have the disadvantage of requiring test materials. Validation of the NRS in children is essential before it can be recommended for use in clinical practice.

Recently, several studies have evaluated the psychometric properties of the NRS in children with acute pain. The NRS showed good convergent, discriminant, 
and criterion validity in a clinical sample of children undergoing surgery. ${ }^{26}$ in addition, the NRS correlated highly with other pain scales, and its distribution of scores was comparable with those of the VAS and FPS-R in children with postoperative pain. ${ }^{37}$ In contrast, a study conducted among children with acute abdominal pain found poor agreement between the verbal NRS and the VAS, colored analog scale, and the Wong-Baker Faces scale. ${ }^{1}$ The NRS was sensitive to change following administration of analgesics, had adequate test re-test reliability, and a high correlation with the VAS in children and adolescents presenting to a hospital emergency department. ${ }^{2}$ As well, a cut-off score of 4 or higher on the 0 to 10 metric of the NRS discriminated between children who, after surgery, perceived themselves as needing versus not needing pain medication whereas a score of higher than 6 discriminated between children who were dissatisfied versus satisfied with treatment. ${ }^{35} \mathrm{Fi}$ nally, in a study comparing pain scales on the first 3 days after pectus excavatum repair, children's verbal NRS scores were higher than their FPS-R and VAS scores. Moreover, the rate of decrease in pain over the 3 postoperative days was significant for the FPS-R and VAS but not the verbal NRS, ${ }^{7}$ suggesting that the latter scale is less responsive to change over time than are the former scales.

Together, these studies have provided valuable psychometric data on the NRS for children with acute pain. In light of the findings described above, ${ }^{7}$ it is especially important to evaluate whether the NRS is sensitive to change ${ }^{9}$ and if it is a valid measure of other dimensions of the pain experience, such as pain unpleasantness. The primary objective of the present study was to evaluate the convergent and discriminant validity of the NRS for pain intensity (I) and unpleasantness (U) in children after surgery. Convergent validity of the NRS is examined by looking at its relationship with other pain scales whereas discriminant validity is evaluated by examining the relationship of the NRS with scales that measure a similar, but nonidentical, construct; in this case, functional disability. Research has shown that the relationship between functional disability and pain levels is not always strong; changes in pain levels are not highly correlated with changes in functional disability, and vice versa. ${ }^{27}$ The present study also examined the sensitivity of the NRS to change over a 2-week period after surgery; its equivalence with other pain measures; and the scale participants liked best and found the easiest to use.

\section{Methods}

\section{Participants and Recruitment}

Children between the ages of 8 and 18 years undergoing either orthopedic (eg, scoliosis, osteotomy, plate insertion tibial/femur, open hip reduction, hip capsulorrhaphy) or general surgical procedures (eg, thoracotomy, thoraco-abdominal surgery, Nuss or Ravitch procedure for pectusexcavatum repair, sternotomy, laparotomy) were eligible to participate in this study. Participants were excluded if they were developmentally or cogni- tively delayed, undergoing surgery for cancer, or not fluent in written and/or spoken English.

\section{Pain Measures}

Three different pain measurement scales were used in this study: NRS, VRS, and faces rating scale (ie, FPS-R and Facial Affective Scale [FAS]). All the pain measures used have evidence of validity in children aged 8 years and older. $^{13,15,17,24,37}$

\section{1-Point Numerical Rating Scale for Pain Intensity (NRSI) and Pain Unpleasantness (NRSU)}

The NRS is a verbally administered scale that measures pain intensity ("how much pain do you feel right now?"). The NRS can also be used to measure pain unpleasantness ("how unpleasant/horrible/yucky is the pain right now?"). The end points represent the extremes of the pain experience. There are no agreed upon NRS anchors for measuring pain intensity and unpleasantness in children. ${ }^{34}$ As such, the following anchors were used in the present study: for pain intensity, $0=$ "no pain at all" to $10=$ "worst possible pain"; for pain unpleasantness, $0=$ "not at all unpleasant/horrible/yucky" to $10=$ "most unpleasant/horrible/yucky feeling possible." Preliminary data suggest that the NRSI has good construct validity in a sample of children with acute postsurgical pain, aged 7 to 17 years. $^{37}$

\section{Verbal Rating Scale for Pain Intensity and Unpleasantness (VRS ${ }^{12}$ )}

The VRS is a 4-point Likert scale designed to measure pain intensity and pain unpleasantness. Patients are asked to choose the best descriptor of the intensity and unpleasantness of their pain at the present moment. For pain intensity, the 4 possible choices are: $0=$ "No pain," 1 = "A little bit of pain," 2 = "A medium amount of pain," 3 = "A lot of pain." For pain unpleasantness, the 4 choices are: $0=$ "Not at all unpleasant/yucky/horrible," 1 = "A little bit unpleasant/yucky/horrible," 2 = "A medium amount unpleasant/yucky/horrible," 3 = "A lot unpleasant/yucky/horrible." The 4-VRS correlates highly with other measures of pain. ${ }^{15}$

\section{Faces Pain Scale-Revised (FPS-R ${ }^{17}$ )}

The FPS- $R$ is a visual scale composed of 6 faces illustrating an increasing level of pain intensity. Children are asked to choose the face that best describes the intensity of the pain they are currently experiencing. Scores range from 0 to 10 with the faces representing the lower and higher levels of pain intensity coded as 0 and 10, respectively. The FPS-R is highly correlated with the visual analog scale $(r=.93)$ and with the colored analog scale $(r=$ .84), showing strong validity. ${ }^{17}$

\section{Facial Affective Scale (FAS ${ }^{22-24}$ )}

The FAS is a self-report, visual scale assessing the extent to which the pain experience is unpleasant. Children are asked to select 1 of 9 faces (of which 4 show 
pain) that best represents how they feel about their pain. The child is told that each face represents how a person would feel "inside" when experiencing pain, and is asked "to point to one of the faces which looks like how you feel down inside - not how your face looks but how you really feel inside." Each face is coded using the affective magnitude ratings ${ }^{22}$ ranging from .04 to .97. The scale has good convergent validity as evidenced by a higher correlation with the colored analog scale for pain unpleasantness than to the colored analog scale for pain intensity. ${ }^{28}$

\section{Functional Disability Inventory (FDI ${ }^{38}$ )}

The Functional Disability Index (FDI) is a 15-item scale that measures difficulties children experience completing specific tasks (eg, "Walking to the bathroom," "Eating regular meals," and "Being at school all day"). The FDI typically uses a 5-point Likert Scale yielding total scores ranging from 0 to 60 . Unintentionally, a 4-point Likert scale was used in this study. Children were asked to rate each item on a scale from 0 (no trouble) to 3 (impossible). Total scores range from 0 to 45 . The FDI has been used with many pediatric populations, including children with chronic pain ${ }^{19,21,30}$ and postsurgical pain. ${ }^{13}$

\section{Procedure}

The study was reviewed and approved by the Research Ethics Boards of the Hospital for Sick Children and York University. Initial contact with potential participants was made by nurses, known to the patients, who were not part of the research team. Potential participants, who expressed an interest in hearing more about the study, were approached by a research team member 48 to 72 hours after surgery. After obtaining informed written parental consent and consent or assent from children, a research team member read to children a set of questionnaires including the NRSI-1, NRSU-1, VRSI-1, VRSU-1, FPS-R, and FAS, and recorded their responses to each item. The order of administration of questionnaires was randomized (http://www.randomization. com) within participants to minimize potential order and fatigue effects. Telephone follow-ups were conducted approximately 2 weeks after discharge from hospital by a research assistant who verbally administered to children the NRSI-2, NRSU-2, VRSI-2, VRSU-2 and FDI. Parents also completed measures, but these results will not be presented here.

\section{Data Analysis}

The validity of the NRS was examined using construct validity (convergent and discriminant validity) as well as responsiveness (sensitivity to change over time). Data were screened for normality; non-normality was addressed using appropriate transformations. Welch statistics were used to analyse transformed variables.

\section{Construct Validity}

Construct validity was examined using convergent validity, discriminant validity, and sensitivity to change over time.

\section{Convergent Validity}

Convergent validity was determined by correlating the NRSI-1 and NRSU- 1 with 2 other scales measuring pain intensity (FPS-R and VRSI-1) and pain unpleasantness (FAS and VRSU-1), using Pearson correlations. Spearman correlations were also examined between the NRSI-2 and VRSI-2; and the NRSU-2 and VRSU-2. Moderate ( $r>$.30) to high $(r>.70)$ correlations $^{18}$ would indicate adequate convergent validity. In addition, the NRSI should correlate more strongly with other measures of pain intensity compared with pain unpleasantness; similarly, the NRSU should correlate more strongly with other measures of pain unpleasantness compared with pain intensity. As such, 2-tailed, paired samples t-tests ${ }^{6}$ were used to compare the strength of the correlation coefficients between the NRSI- 1 and FPS-R compared with NRSI- 1 and FAS and between the NRSU-1 and FAS compared with NRSU-1 and FPS-R. The strength of the correlation coefficients between the NRSI- 1 and VRSI- 1 compared with the NRSI-1 and VRSU- 1 and between the NRSU- 1 and VRSU- 1 compared with NRSU-1 and VRSI- 1 were also examined using a t-statistic. ${ }^{6}$ A t-statistic was used to account for the lack of independence between correlation coefficients (ie, the NRS is part of both correlation coefficients being compared).

\section{Discriminant Validity}

Discriminant validity was determined by correlating the NRS with scores of functional disability. Research has shown that the relationship between functional disability and pain levels is not always strong; changes in pain levels are not highly correlated with changes in functional disability, and vice versa. ${ }^{27}$ As such, moderate correlations $(.30<r>.70)$ between the NRSI-1, NRSU-1, NRSI-2, and NRSU-2 with the FDI would indicate adequate discriminant validity.

\section{Sensitivity to Change Over Time}

Pearson correlations were examined between the NRSI- 1 and NRSI-2 and between the NRSU-1 and NRSU-2. Paired samples t-tests were used to compare changes in mean pain intensity (NRSI) and mean pain unpleasantness (NRSU) ratings from 48 to 72 hours after surgery to 2 weeks after surgery. It was expected that pain intensity and pain unpleasantness scores 2 weeks after surgery would be lower than their respective inhospital scores. As such, significantly lower scores at the 2-week assessment would indicate adequate ability of the NRS to detect changes in acute pain levels over time. The sensitivity to change over time of the VRS (VRSI-1 with VRSI-2 and VRSU-1 with VRSU-2) was also examined. Given that the VRS is an ordinal scale, Wilcoxon ranked signed test was used. Because the NRS is scored on an 11-point rating scale and the VRS is scored on a 4-point scale, it was expected that the NRS would be more sensitive to change over time. The NRS is a continuous measure whereas the VRS is considered as ordinal and, as such, no direct statistical procedures can be used to compare their sensitivity to change. 


\section{Equivalence of Pain Scales}

In order to examine the equivalence of pain scales, absolute pain score differences between the NRSI-1 and FPS-R were examined. Both the NRSI and the FPS-R are scored on a scale from 0 to 10 . If children's pain reports on these 2 scales are equivalent, then the pain score difference should be equal to zero. Score differences for pain unpleasantness were not examined given that the NRSU is an 11-point scale and the FAS uses 9 faces of which 4 show pain (range of scores .04 through .97) and, as such, direct comparisons between the scales cannot be made.

We also examined the equivalence of the VRS and NRS by comparing the NRSI-1, NRSU-1, NRSI-2, and NRSU-2 across levels of the VRSI-1, VRSU-1, VRSI-2, and VRSU-2, respectively. It was expected that scores on the NRSI-1 would increase significantly from 1 category to another on the VRSI-1 (eg, NRS scores corresponding to the VRS category "a little bit of pain" would be lower when compared with those corresponding to the category "a medium amount of pain"). A series of 4 Bonferronicorrected $(\alpha=.0125), 1$-way univariate analysis of variance (ANOVA) tests was performed using the 4 levels of the VRS scale as the factor and the participant's corresponding NRS score as the dependent variable. Significant main effects were explored with multiple comparisons using Bonferroni corrections. When the assumption of homogeneity of variance was violated, Welch F-statistics and Games-Howell post hoc tests were used.

\section{Children's Preferences for Pain Scales and Their Ease of Use}

After completing the NRSI-1, VRSI-1, and FPS-R, children were asked: 1) "Which one of the three scales did you find easiest to do? And which one did you find hardest to do?" 2) "Which one did you like doing best? And which one did you like doing least?" and 3) "Are there any other things about the three ways of measuring the pain you feel that you would like to tell us?" Children also answered the same questions after completing the pain unpleasantness scales (NRSU-1, FAS, and VRSU-1). Multinomial logistic regression analyses were used to examine if age and gender significantly influenced which pain scale children liked best and found easiest to use. Chi-square Goodness of Fit was used to examine differences in children's opinions of ease of use and likability of pain scales.

\section{Sample Size Estimation}

Sample size was calculated based on the primary objective of this study (to examine the convergent and discriminant validity of the NRS for both pain intensity [I] and unpleasantness [U]). As such, sample size was calculated a priori for Pearson correlations using G*Power v.3.1.2. ${ }^{11}$ Sample size analysis showed that 84 participants would be required to find a significant correlation of $r=.30$ at $\alpha=.05$ (2-tailed) and power $=80 \%$. All analyses were conducted using SPSS v. 19.0 .

\section{Results}

Skewness and kurtosis significance testing (estimate/ standard error $>3$ ) did reveal non-normality for the FAS, NRSI-2, and NRSU-2. Exponential transformation was used on the FAS to achieve normality and square root transformation was used on both the NRSI-2 and NRSU-2. All subsequent analyses are performed using these 3 transformed variables (FAS ${ }^{t}, N$ RSI- $2^{t}$, and NRSU$\left.2^{t}\right)$ unless otherwise specified.

\section{Recruitment}

Recruitment took place between July 2008 and September 2010 . Of the 4,054 children who underwent orthopaedic or general surgery during this time period, 3,150 were excluded because they did not undergo 1 of the 11 surgeries included in this study and another 370 children were excluded because they were younger than 8 years of age. Another 131 potential participants were excluded because they had cognitive or developmental delay $(n=34)$, were noncommunicative ( $n=$ 31 ), had severe cerebral palsy ( $n=44)$, their parents were not fluent in written and spoken English ( $n=19)$, had cancer $(n=2)$ or had congenital insensitivity to pain $(n=1)$. Among the 403 eligible participants, 255 were not approached due to time constraints or logistical reasons (already discharged [ $=71]$, in the PICU $[n=21]$, sleeping [ $n=22]$, parents absent $[n=40]$, undergoing additional medical procedures [ $n=6]$, or research staff not available [ $n=95]$ ). Of the 148 eligible participants who were approached and asked to participate, 65 (43.9\%) declined. A total of 83 children took part in this study and $83 \%$ of them $(n=69)$ completed the 2 -week telephone follow-up (mean number of days from the initial assessment $=15.62, \mathrm{SD}=2.2$ ).

\section{Descriptive Statistics}

A total of 83 children (girls $=56[67.5 \%]$ ) between the ages of 8 and 18 years ( mean $=13.8, S D=2.4$ ) and 1 of their parents (mothers $=63[75.9 \%]$, mean age $=43.4$, $\mathrm{SD}=5.9 ;$ fathers $=20$ [24.1\%]; mean age $=46.1, \mathrm{SD}=$ 8.2) participated in this study. The ethnicity of participants included Caucasian (64\%), Asian (12\%), AfricanCaribbean or African-Canadian (8.4\%), Middle Eastern $(4.8 \%)$, and "other" or unspecified $(7.2 \%)$. English was the first language spoken at home for the majority of children (89\%). Most parents had at least a college/undergraduate university degree $(\mathbf{7 4 \% )}$. Almost one-third of parents $(31.7 \%)$ reported currently experiencing pain problems while one-quarter of all parents $(24.4 \%)$ reported past or current chronic pain (pain that lasted for 3 months or longer). Most parents with ongoing pain problems $(75 \%)$ also reported experiencing past/ current chronic pain.

The children in this sample underwent surgery for scoliosis (spinal fusion) ( $n=42,50.6 \%$ ), osteotomy $(n=25$, $30.1 \%)$, Nuss or Ravitch procedure for pectus excavatum repair $(n=8,9.6 \%)$, laparotomy $(n=7,8.4 \%)$, and thoracotomy $(n=1,1.2 \%)$. Approximately half of the children had never had surgery in the past $(n=44,53 \%$ ) 
whereas $39(47 \%)$ had undergone, on average, 2 $(\mathrm{SD}=1.6)$ surgical interventions in the past (range $=$ $1-7)$. The majority of children retrospectively reported no pain $(n=36,43.4 \%)$ or a little pain $(n=31,37.3 \%)$ prior to the present surgery, while the remaining participants reported a medium amount $(n=13,15.7 \%)$ or a lot $(n=3,3.6 \%)$ of pain. Significant differences were not found in elapsed time from initial assessment to follow-up $(P=.160)$ or in pain intensity or pain unpleasantness scores 48 to 72 hours after surgery (NRSI: $P=$ .217; NRSU: $P=.355$ ) and 2 weeks after discharge (NRSI: $P=.078$; NRSU: $P=.260$ ) across the different types of surgical procedures.

Chi-square test revealed significant gender differences across types of surgical procedures $(\chi 2=23.25, P<.001)$ in that fewer boys had surgery for scoliosis than expected and more boys had a Nuss or Ravitch procedure than expected.

Mean total scores and standard deviations (median and range were used for the ordinal VRS scale) on the pain scales measured 48 to 72 hours after surgery and 2 weeks after discharge from hospital are presented in Table 1. Compared with boys, girls reported significantly higher levels of pain unpleasantness 48 to 72 hours after surgery (NRSU-1: mean score girls $=4.98 \pm$ SD, mean score boys $=3.70 \pm$ SD) and functional disability 2 weeks after discharge from hospital (FDI: mean score girls = $21.15 \pm$ SD, mean score boys = $16.24 \pm$ SD). Girls and boys did not differ significantly on any other measures. Correlation coefficients presented in Table 2 show that age was not significantly correlated with functional disability or any of the measures of pain intensity or unpleasantness (all $P>.05$ ).

\section{Construct Validity}

\section{Convergent Validity}

As shown in Table 2, the NRSI-1 and NRSU-1 correlated significantly with the FPS-R and VRSI-1 and the FAS $^{t}$ and VRSU-1, respectively. The NRSI- ${ }^{t}$ and NRSU$2^{t}$ significantly correlated with the VRSI-2 and VRSU-2, respectively.

The NRSI- 1 correlated significantly more with the FPS-R (another measure of pain intensity) than it did with the FAS $^{t}$ (a measure of pain unpleasantness) ( $t[1,80]=$ 2.68, $P=.01$ ). The NRSI-1 also correlated significantly more with the VRSI-1 than it did with the VRSU-1 (t [1, $80]=3.41, P<.01)$. Similarly for pain unpleasantness, the NRSU-1 correlated significantly more strongly with the FAS ${ }^{t}$ than it did with the FPS-R $(t[1,80]=2.23, P=$ $.03)$. The NRSU-1 also correlated significantly more strongly with the VRSU-1 than it did with the VRSI-1 $(t[1,80]=2.38, P=.02)$.

\section{Discriminant Validity}

As shown in Table 2, significant, moderate correlations were found between pain intensity and unpleasantness 48 to 72 hours and 2 weeks after discharge from hospital and functional disability (FDI) measured 2 weeks after discharge from hospital.
Table 1. Descriptives of Pain and Functional Disability Scales

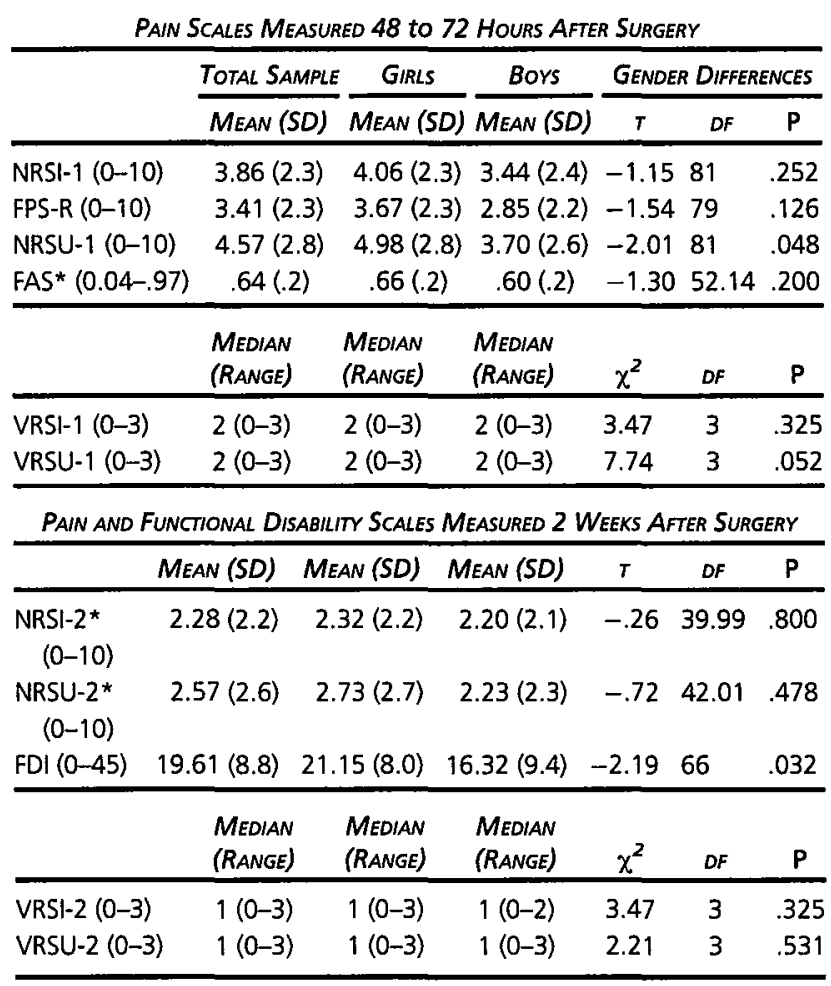

Abbreviations: NRSI-1, Numerical Rating Scale for Pain Intensity measured 48 to 72 hours after surgery; VRSI-1, Verbal Rating Scale for Pain Intensity measured 48 to 72 hours after surgery; FPS-R, Faces Pain Scale Revised measured 48 to 72 hours after surgery; NRSU-1, Numerical Rating Scale for Pain Unpleasantness measured 48 to 72 hours after surgery; VRSU-1, Verbal Rating Scale for Pain Unpleasantness measured 48 to 72 hours after surgery; FAS, Facial Affective Scale measured 48 to 72 hours after surgery; NRSI-2, Numerical Pain Rating Scale measured approximately 2 weeks after discharge from hospital; VRSI-2, Verbal Rating Scale for Pain Intensity measured approximately 2 weeks after discharge from hospital; NRSU-2, Numerical Rating Scale for Pain Unpleasantness measured approximately 2 weeks after discharge from hospital; VRSU-2, Verbal Rating Scale for Pain Unpleasantness measured approximately 2 weeks after discharge from hospital; FDI, total score on the Functional Disability Inventory measured approximately 2 weeks after discharge from hospital (in this study, the Functional Disability Inventory was measured using a Likert scale ranging from 0 [no trouble] to 3 [impossible]).

*Transformed variables were used for the FAS, NRSI-2 and NRSU-2 to examine gender differences using Welch t-statistics (original scores are presented for means and standard deviations.

\section{Sensitivity to Change Over Time}

Correlation coefficients between the NRS and VRS 48 to 72 hours after surgery and 2 weeks after discharge are shown in Table 2 . Using the nontransformed NRSI2 and NRSU-2 data, results showed that scores on the NRSI-1 and NRSU-1 were significantly higher than scores on the NRSI-2 (mean change $=1.49 ; \mathrm{t}[68]=5.25 ; P<$ .001 ; effect size $=.68$ ) and NRSU-2 (mean change $=$ $1.89 ; t[68]=5.30 ; P<.001$; effect size $=.72$ ), respectively. In addition, significant differences were found between the VRSI- 1 and VRSI-2 $(z=-4.272, P<.001)$ as well as between the VRSU-1 and VRSU-2 $(z=-3.638, P<$ $.001)$. As such, both the NRS and VRS for pain intensity and unpleasantness showed a significant decrease from 48 to 72 hours to 2 weeks after discharge from hospital. 
Table 2. Correlation Coefficients and Partial Correlations Controlling for Age and Gender on Various Pain Scales

\begin{tabular}{|c|c|c|c|c|c|c|c|c|c|c|c|c|}
\hline & 1 & 2 & 3 & 4 & 5 & 6 & 7 & 8 & 9 & 10 & 11 & 12 \\
\hline \multicolumn{13}{|l|}{ 1.AGE } \\
\hline 2.FPS-R & .20 & & $.73^{* *}$ & $.70 * *$ & $.55^{* *}$ & $.64^{* *}$ & $.60^{* *}$ & $.32 * *$ & .12 & $.41 * *$ & .22 & $.48^{* *}$ \\
\hline 3.NRSI-1 & .19 & $.76^{* *}$ & & $.70 * *$ & $.51 * *$ & $.66^{* *}$ & $.40 * *$ & $.45^{* *}$ & .20 & $.56^{\star \star \star}$ & $.28^{*}$ & $.41^{\star *}$ \\
\hline 4.VRSI-1† & .17 & $.71 * \star$ & $.75^{\star \star}$ & & $.46 * \star$ & $.50 \star \star$ & $.52^{\star \star}$ & $.28^{\star}$ & .18 & $.43^{\star \star}$ & $.30^{*}$ & $.29^{*}$ \\
\hline 5.FAS ${ }^{t}$ & .11 & $.57^{\star \star}$ & $.51 * \star$ & $.49 * \star$ & & $.71 * *$ & $.58^{\star \star}$ & $.32 * \star$ & $.27^{*}$ & $.40 * *$ & $.35 * *$ & $.29^{*}$ \\
\hline 6.NRSU-1 & .07 & $.68^{* *}$ & $.66^{* *}$ & $.49 * *$ & $.71 * *$ & & $.61 * *$ & $.29^{\star}$ & -.06 & $.38^{* *}$ & .16 & $.29^{*}$ \\
\hline 7.VRSU-1† & -.02 & $.57^{* *}$ & $.40^{* *}$ & $.47^{* *}$ & $.54^{* *}$ & $.67^{* \star}$ & & $.27^{*}$ & .09 & $.34^{\star \star}$ & .23 & $.28^{\star}$ \\
\hline 8.NRSI-2 ${ }^{t}$ & .05 & $.32^{\star \star}$ & $.45^{\star \star}$ & $.28^{\star}$ & $.32^{\star \star}$ & $.30 *$ & .24 & & $.82^{\star \star}$ & $.84^{* *}$ & $.75 * *$ & $.52^{* *}$ \\
\hline 9.VRSI-2† & .12 & $.27^{*}$ & $.35^{\star \star}$ & $.28 *$ & $.31 *$ & .18 & .24 & $.80 * *$ & & $.76^{* *}$ & $.72 * *$ & $.31^{*}$ \\
\hline 10.NRSU-2 ${ }^{t}$ & .01 & $.41 * *$ & $.56^{* *}$ & $.41^{\star *}$ & $.40^{\star \star}$ & $.39 * *$ & $.30^{*}$ & $.84^{* *}$ & $.77 * *$ & & $.784^{*}$ & $.51^{* *}$ \\
\hline 11.VRSU-2† & .05 & $.32^{\star *}$ & $.38^{* *}$ & $.35^{\star *}$ & $.36^{* *}$ & $.30^{*}$ & $.30^{*}$ & $.75^{\star *}$ & $.75^{\star \star}$ & $.83^{\star \star}$ & & $.38^{\star \star}$ \\
\hline 12.FDI & -.11 & $.43^{\star \star}$ & $.43^{\star \star}$ & $.29^{\star}$ & $.29^{\star}$ & $.35^{\star \star}$ & $.33^{\star \star}$ & $.49 * \star$ & $.41 \star \star$ & $.50 * *$ & $.38 * \star$ & \\
\hline
\end{tabular}

Abbreviations: FPS-R, Faces Pain Scale Revised measured 48 to 72 hours after surgery; NRSI-1, Numerical Rating Scale for Pain intensity measured 48 to 72 hours after surgery; VRSI-1, Verbal Rating Scale for Pain Intensity measured 48 to 72 hours after surgery; FAS', Facial Affective Scale measured 48 to 72 hours after surgery (exponential transformation); NRSU-1, Numerical Rating Scale for Pain Unpleasantness measured 48 to 72 hours after surgery; VRSU-1, Verbal Rating Scale for Pain Unpleasantness measured 48 to 72 hours after surgery; NRSI-2t Numerical Pain Rating Scale measured approximately 2 weeks after discharge from hospital (square root transformation); VRSI-2, Verbal Rating Scale for Pain Intensity measured approximately 2 weeks after discharge from hospital; NRSU-2t, Numerical Rating Scale for Pain Unpleasantness measured approximately 2 weeks after discharge from hospital (square root transformation); VRSU-2, Verbal Rating Scale for Pain Unpleasantness measured approximately 2 weeks after discharge from hospital; FDI, total score on the Functional Disability Inventory measured approximately 2 weeks after discharge from hospital (in this study, the Functional Disability Inventory was measured using a Likert scale ranging from 0 [no trouble] to 3 [impossible]).

NOTE. Pearson correlation coefficients are displayed below the diagonal while partial correlation coefficients controlling for age and gender are displayed above the diagonal.

*indicates significance at the $P=0.05$ level.

†Spearman correlation coefficients were used below the diagonal for the VRSI-1, VRSU-1, VRSI-2 and VRSU-2.

**indicates significance at the $P=0.01$ level.

\section{Equivalence of Pain Scales}

As shown in Fig 1, $55(68 \%)$ of children rated their pain within a 1-point difference on the NRSI and FPS-R, while there was a 2-point difference or less for $71(88 \%)$ children and a 3-point difference or less for $78(96 \%)$ children. A 4-point difference or more was only found in 3 children. Age was not significantly correlated with the distribution of differences in pain scores between the NRSI and FPS-R. There were also no gender differences in the distribution of differences in pain scores between the NRSI and FPS-R (t $[79]=-.092, P=.927)$.

Table 3 shows that for both pain intensity and pain unpleasantness measured 48 to 72 hours after surgery and 2 weeks after discharge, scores on the NRS increased significantly as scores on the VRS increased (from "No pain" to "A little bit of pain"; to "A medium amount of pain"; to "A lot of pain"). The 1 exception to this pattern was that for the NRSI-2t, significant differences were not found between "A medium amount of pain" and "A lot of pain". Fig 2 shows box plots of (untransformed) NRS scores across categories of the VRS for both pain intensity and unpleasantness.

\section{Evaluation of Children's Preferences for Pain Scales and Their Ease of Use}

Table 4 depicts children's evaluation of pain scales to measure both pain intensity and unpleasantness. Results indicate that for pain intensity, the FPS-R was the bestliked scale whereas the VRS was the least-liked scale. In terms of ease of use, the FPS-R was rated as the easiest whereas none of the 3 scales significantly stood out as the hardest. The pattern of findings for pain intensity and pain unpleasantness scales was similar (Table 4).
Overall, as shown in Table 5, children liked the FPS best, primarily because they reported that it better described the pain they felt. The VRS was the least-liked scale mainly because it did not offer enough choices to describe their pain. Children who liked the NRS best, reported that they were more familiar with this type of scale and found numbers easier to use when quantifying their pain. There were no significant effects of age or gender on children's best-liked scale, least-liked scale,

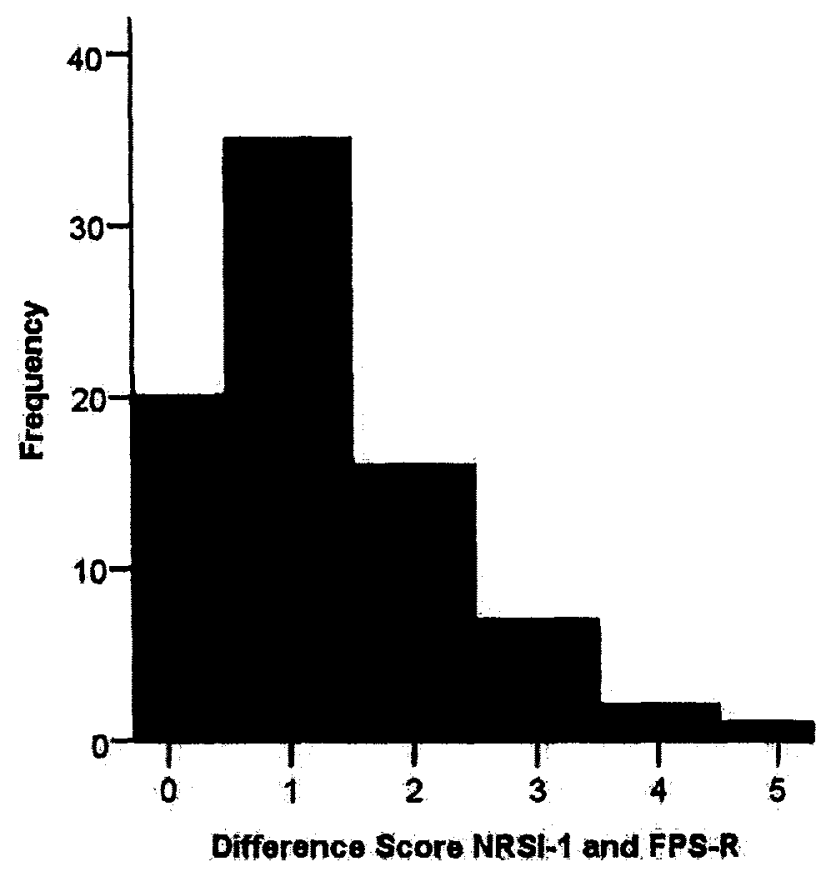

Figure 1. Histogram of absolute values of score differences between the NRSI-1 and FPS-R. 
Table 3. One-Way Anovas Comparing Scores on the NRS Across Levels of the VRS

\begin{tabular}{|c|c|c|c|c|c|c|}
\hline & \multirow[b]{2}{*}{$F$} & \multirow[b]{2}{*}{$D F$} & \multirow[b]{2}{*}{$\mathbf{P}$} & \multicolumn{3}{|c|}{ MuLtIPLE COMPARISONS USING BONFERRONI CORRECTION } \\
\hline & & & & & MEAN DIFFERENCE & $\mathbf{P}$ \\
\hline \multicolumn{7}{|c|}{ PaIN INTENSTYY } \\
\hline \multicolumn{7}{|c|}{ VRSI } \\
\hline \multirow[t]{3}{*}{ NRSI-1 } & 33.77 & 3,79 & $<.001$ & No pain vs a little bit of pain & -2.085 & .011 \\
\hline & & & & A little bit of pain vs a medium amount of pain & -2.101 & $<.001$ \\
\hline & & & & A medium amount of pain vs a lot of pain & -2.015 & .002 \\
\hline \multirow[t]{5}{*}{ NRSI- $2^{t}$} & 46.67 & 3,65 & $<.001$ & No pain vs a little bit of pain & -1.129 & $<.001$ \\
\hline & & & & A little bit of pain vs a medium amount of pain & -.846 & $<.001$ \\
\hline & & & & A medium amount of pain vs a lot of pain & -.507 & .726 \\
\hline & & & & PaIN UnPLEASANTNESS & & \\
\hline & & & & VRSU & & \\
\hline \multirow[t]{3}{*}{ NRSI-1 } & 24.99 & 3,79 & $<.001$ & Not at all unpleasant vs a little bit unpleasant & -2.983 & .002 \\
\hline & & & & A little bit unpleasant vs a medium amount unpleasant & -1.743 & .008 \\
\hline & & & & A medium unpleasant vs a lot unpleasant & -2.241 & .004 \\
\hline \multirow[t]{3}{*}{ NRSU- $2^{t *}$} & 482.55 & $3 ; 27.9$ & $<.001$ & Not at all unpleasant vs a little bit unpleasant & -1.369 & $<.001$ \\
\hline & & & & A little bit unpleasant vs a medium amount unpleasant & -.605 & .001 \\
\hline & & & & A medium unpleasant vs a lot unpleasant & -.779 & .018 \\
\hline
\end{tabular}

Abbreviations: NRSI-1, Numerical Rating Scale for Pain Intensity measured 48 to 72 hours after surgery; NRSI- ${ }^{\mathrm{t}}$, Numerical Rating Scale for Pain Intensity measured 2 weeks after discharge (square root transformation); NRSU-1, Numerical Rating Scale for Pain Unpleasantness measured 48 to 72 hours after surgery; NRSU- ${ }^{\mathrm{t}}$, Numerical Rating Scale for Pain Unpleasantness measured 2 weeks after discharge (square root transformation); VRSU-2, Verbal Rating Scale.

*Indicate that Welch F-statistic and Games-Howell post hoc tests were used to adjust for violation of the assumption of homogeneity of variance.

scale they found easiest, or scale they found hardest to use $(P=.416-.942)$.

\section{Discussion}

The goal of this study was to provide additional support for the validity of the NRS (for pain intensity and pain unpleasantness) in children after major surgery. Re- sults showed that the NRS for pain intensity and pain unpleasantness has good convergent validity (correlated highly with the VRSI and VRSU as well as FPS-R and FAS, respectively); discriminant validity (moderate correlations with the FDI); and sensitivity to change over a 2-week period. Results of the 1-way ANOVA suggested that the NRS can be used interchangeably with the VRS, and examination of score differences between the NRS
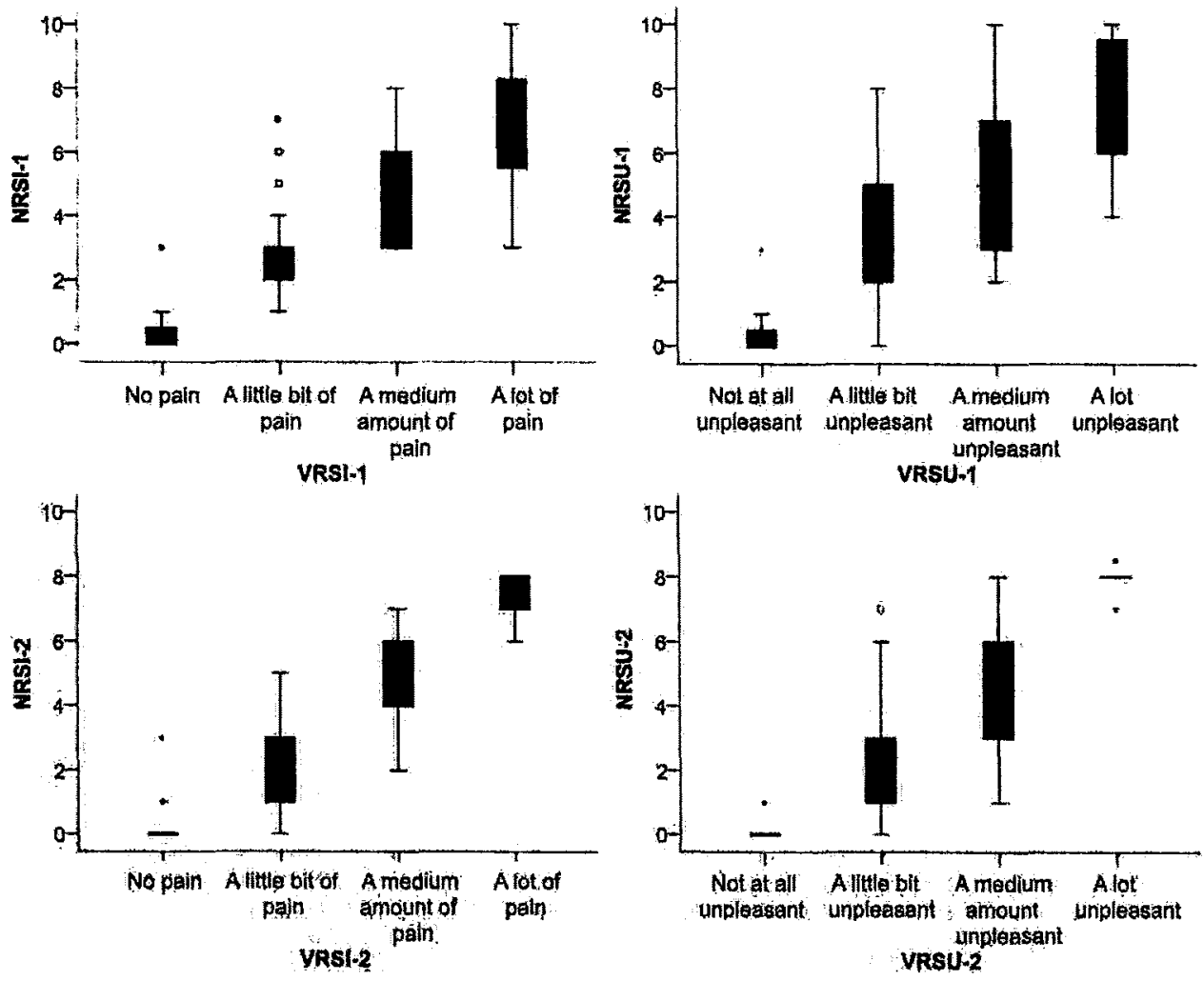

Figure 2. Boxplot of NRS scores across levels of VRS. 
Table 4. Chi-Square Goodness of Fit Test Examining Children's Preferred Measurement Tool to Assess Pain Intensity and Unpleasantness

\begin{tabular}{|c|c|c|c|c|c|c|}
\hline \multicolumn{7}{|c|}{ PAIN INTENSITY } \\
\hline & NRSI & FPS-R & VRSI & $x^{2}$ & $D F$ & $\mathbf{P}$ \\
\hline \multicolumn{7}{|l|}{ Liked best } \\
\hline $\mathrm{n}$ & 27 & 33 & 9 & 13.565 & 2 & .0011 \\
\hline Residuals & .834 & 2.085 & -2.919 & & & \\
\hline \multicolumn{7}{|l|}{ Liked least } \\
\hline$n$ & 19 & 20 & 30 & 3.22 & 2 & .200 \\
\hline Residuals & -.834 & -.626 & 1.459 & & & \\
\hline \multicolumn{7}{|c|}{ Easiest to use } \\
\hline$n$ & 28 & 35 & 8 & 16.592 & 2 & .0002 \\
\hline Residuals & .891 & 2.330 & -3.220 & & & \\
\hline \multicolumn{7}{|c|}{ Hardest to use } \\
\hline $\mathrm{n}$ & 24 & 29 & 16 & 3.739 & 2 & .154 \\
\hline Residuals & .209 & 1.251 & -1.460 & & & \\
\hline \multicolumn{7}{|c|}{$P_{A I N} U_{N P L E A S A N T N E S S}$} \\
\hline & NRSU & FAS & VRSU & $x^{2}$ & $D F$ & $P$ \\
\hline \multicolumn{7}{|l|}{ Liked best } \\
\hline$n$ & 28 & 38 & 4 & 26.171 & 2 & .0000 \\
\hline Residuals & .966 & 3.036 & -4.002 & & & \\
\hline \multicolumn{7}{|l|}{ Liked least } \\
\hline $\mathrm{n}$ & 16 & 17 & 36 & 11.043 & 2 & .0040 \\
\hline Residuals & -1.460 & -1.251 & 2.711 & & & \\
\hline \multicolumn{7}{|c|}{ Easiest to use } \\
\hline $\mathrm{n}$ & 26 & 40 & 3 & 30.348 & 2 & .0000 \\
\hline Residuals & .626 & 3.546 & -4.170 & & & \\
\hline \multicolumn{7}{|c|}{ Hardest to use } \\
\hline$n$ & 19 & 17 & 32 & 5.853 & 2 & .0536 \\
\hline Residuals & -.770 & -1.190 & 1.960 & & & \\
\hline
\end{tabular}

Abbreviations: NRSI, Numerical Rating Scale for Pain Intensity; VRSI, Verbal Rating Scale for Pain Intensity; FPS-R, Faces Pain Scale Revised; NRSU, Numerical Rating Scale for Pain Unpleasantness; VRSU, Verbal Rating Scale for Pain Unpleasantness; FAS, Facial Affective Scale.

NOTE. $+2<$ residuals $<-2$ represent the cut-off for significance.

and FPS-R suggested that a small variation in pain scores (<3) exists between the 2 scales. Results also suggest that the NRS is as valid to measure pain unpleasantness as it is to measure pain intensity.

Interestingly, both the NRS and VRS demonstrated statistically significant decreases from 48 to 72 hours postsurgery to the 2-week follow-up. These results are in contrast to those from the adult literature which suggest that the NRS has greater sensitivity and that the VRS has inadequate sensitivity. ${ }^{39} \mathrm{~A}$ review of pain rating scales has shown that the NRS might be one of the most sensitive scales to detect changes in pain levels in adults; ${ }^{39}$ the more response options a scale possesses, the more sensitive it tends to be. This suggests that while the NRS offers more response options compared with the VRS, the latter might offer more concrete and specific categories for children to rate their pain experience.

The results of the present study also are not consistent with those from a recent study which found that the verbally administered NRS, in contrast with other pain measures, did not decrease over the first 3 days after surgery. ${ }^{7}$ It is possible that this discrepancy in findings is due to the difference in time elapsed between measurements ( 2 days $^{7}$ versus 2 weeks in the present study) and that the NRS lacks sensitivity to smaller changes in pain intensity across a shorter time period. It is also possible that results from Connelly and Neville's study ${ }^{7}$ reflect a response bias in that participants might have remembered the number they reported in the previous assessment that took place only 2 hours prior. It is possible that it was easier for participants to remember the number they reported in the previous assessment than the face they picked or where on a continuous scale they had rated their pain. It would be interesting for future studies to examine whether the NRS is sensitive to change in pain intensity over time in the acute postoperative period by measuring NRS pain scores only once a day and as such minimizing recall bias. Such study would use an alternative pain scale for clinical purposes to ensure adequate pain assessment, but would make it possible to further evaluate the sensitivity to change of the NRS.

In addition, the minimum clinically significant difference of the NRS in pediatric populations has not been clearly established. Research conducted in musculoskeletal pain ${ }^{31}$ and emergency department ${ }^{20}$ adult samples has found that approximately a 1-point change on the NRS is associated with "a little more/less pain" while a 2-point decrease on the NRS is associated with a report of "much better." It is unclear however, if these minimum clinically significant differences on the NRS also apply to pediatric postsurgical patients. Only 2 studies have examined how changes in NRS scores correspond with minimum clinically meaningful changes in the pain experience of children. ${ }^{2,35}$ Both studies suggested that a 1 point difference on the 0 to 10 NRS correspond with "a little better" or "a little worse" pain. What is not clear however is whether a 1-point change on the NRS translates into a need to intervene clinically (eg, a need for more or less pain medication). In the present study, the mean change scores (from 24 to 48 hours postop to the 2-week follow-up) were 1.49 (15\% decrease on the NRS) for pain intensity and 1.89 (19\% decrease on the NRS) for pain unpleasantness. These differences would exceed the minimum clinically significant difference in pain scores of 1 point described above. ${ }^{2,35}$ These results are also consistent with the minimum clinically significant difference established for the VAS in children (a change of $10 \mathrm{~mm}$ or $10 \%$ ). ${ }^{29}$ It will be important for future research to validate the minimum clinically significant difference on the NRS across various pediatric patient populations. Establishing the minimum clinically significant difference on the NRS is important, but it would only provide general guidelines that have little specificity in terms of clinical decision making. ${ }^{8}$ Finding a cut-off score that indicate the need for pain management interventions would not take into account individuality and differences in the ways each child reports his/her pain.

A gender difference was found for pain unpleasantness 48 to 72 hours after surgery using the NRSU. This difference was not evident for the VRSU, FAS, nor any of the 3 pain intensity scales. Whereas it is possible that there is a gender difference in pain unpleasantness that only the 
Table 5. Qualitative Examination of Children's Preferences for Pain Measurement Tools

\begin{tabular}{|c|c|c|}
\hline \multicolumn{3}{|c|}{ PaIN INTENSITY } \\
\hline & Positive AsPeCtS & NegATIVE Aspects \\
\hline NRSI & $\begin{array}{l}\text { NRSI is most familiar and pain is easier } \\
\text { to quantify with numbers } \\
\text { Numbers have a greater range of response; } \\
\text { more precise } \\
\text { Numbers allow you to avoid talking about the pain }\end{array}$ & Numbers are too variable from 1 person to the other \\
\hline FPS-R & $\begin{array}{l}\text { Faces scale is more expressive. It describes } \\
\text { feelings better } \\
\text { Faces scale is more natural }\end{array}$ & $\begin{array}{l}\text { Faces are too subjective; don't discriminate enough; confusing } \\
\text { Need to look at the scale and it's too tiring } \\
\text { after surgery; takes too long } \\
\text { Not enough faces to choose from } \\
\text { It's difficult to see the pain in each face } \\
\text { Words don't offer enough choices; too vague }\end{array}$ \\
\hline \multicolumn{3}{|c|}{ PAIN UNPLEASANTNESS } \\
\hline & Positive AsPects & NEGATIVE ASPECTS \\
\hline NRSU & $\begin{array}{l}\text { NRSI is most familiar and pain is easier to quantify } \\
\text { with numbers } \\
\text { Number have a greater range of response; } \\
\text { more precise } \\
\text { Numbers allow you to avoid talking about the pain }\end{array}$ & \\
\hline FAS & $\begin{array}{l}\text { Faces scale is more expressive. It describes } \\
\text { feelings better } \\
\text { Faces scale is more natural } \\
\text { Faces are more fun to answer }\end{array}$ & $\begin{array}{l}\text { Faces are too subjective; don't discriminate enough; confusing } \\
\text { Need to look at the scale and it's too tiring after surgery; } \\
\text { takes too long } \\
\text { It's difficult to see the pain in each face }\end{array}$ \\
\hline VRSU & Words describe better than numbers & Words don't offer enough choices; too vague \\
\hline
\end{tabular}

Abbreviations: NRSI, Numerical Rating Scale for Pain Intensity; VRSI, Verbal Rating Scale for Pain Intensity; FPS-R, Faces Pain Scale Revised; NRSU, Numerical Rating Scale for Pain Unpleasantness; VRSU, Verbal Rating Scale for Pain Unpleasantness; FAS, Facial Affective Scale.

NOTE. Some children reported they found all 3 pain intensity scales $(n=7)$ and pain unpleasantness scales $(n=6)$ to be equivalent in terms of ease of use and likeness.

NRS was able to detect, the absence of a difference between boys and girls on the other measures is not consistent with pediatric ${ }^{14}$ and adult ${ }^{32}$ patient samples showing that females score higher than males on pain unpleasantness. It is also possible, however, that this result represents a difference in the way girls use numbers to quantify pain unpleasantness compared with boys and as such the observed difference on the NRSU could be attributed to the type of scale used (ie, numerical). Another possibility is that the difference observed in pain unpleasantness ratings is an artifact of the gender difference observed in the type of surgical procedures underwent by girls and boys. If this were the case though, one would also have expected similar differences in pain intensity scores.

Children favored the faces scales (FPS-R and FAS) over the VRS in terms of likability and ease of use for both pain intensity and unpleasantness. Findings suggest that the VRS was liked best by the fewest number of participants and liked least by the most, indicating that it is not the preferred scale. There were no significant gender or age differences in children's preferences for pain scales or for the scale they found easiest or hardest to complete, suggesting that the same scale could be used across age groups and genders. These results contrast with those of a previous study which found that even though all age groups and both genders tended to prefer the FPS-R, this tendency was more pronounced for girls and younger children. ${ }^{26}$
Consistent with the findings from Miró et al ${ }^{26}$ as well as von Baeyer et $\mathrm{al}^{37}$ the present results did not show an association of age with NRS pain intensity ratings, or differences in ratings across pain measures in children of 8 years of age and older. These results suggest that the NRS has adequate validity across age groups. It is important, however, to keep in mind when choosing to use the NRS in younger children that the child's ability to reason numerically is essential to valid self-reports, and that no one scale will be optimal across child age groups., ${ }^{5,34}$ it is important to examine whether the verbally administered NRS can also be used by younger children, including 6and 7-year-olds. Although the NRS has been validated in children as young as 6 years old, ${ }^{26}$ future validation studies of the NRS in young children should examine their cognitive ability to understand quantitative information and their tendency to select the extreme numbers on the NRS compared with other scales (eg, FPS-R). ${ }^{26}$ Young children also tend to provide answers to questions regardless of their understanding and such bias can only be determined with multiple testing. ${ }^{36}$

There are several limitations to this study. First, this study assessed pain-related measures only after surgery, but not before. It was thus not possible to examine sensitivity of the NRS to change from baseline to postsurgery. In addition, the sample size did not allow for an examination of the validity of the NRS in each age group separately. The $17 \%$ attrition rate from the initial assessment to the follow-up might have led to a reduction in 
the power to detect effects at the follow-up and as such, with no attrition at the follow-up, we might have found even larger correlation coefficients. Furthermore, a 4point scale was inadvertently used for the FDI instead of the standard 5-point scale. It is possible that the reduced variation in responses may have influenced the observed relationship with pain scales. Additional studies should replicate these findings using a 5-point FDI scale. Lastly, the NRS was administered verbally, while the FPS-R and FAS relied on a visual component. It will be important for future studies to compare the validity of a written administration of the NRS with the verbally administered NRS (also referred to as the Verbal Numerical Scale ${ }^{1,2}$ ) as well as with the FPS-R and FAS to rule out any potential administration effects.

\section{Conclusion}

Notwithstanding these limitations, the results from this study add to the emerging literature on the psychometric properties of the NRS in pediatric populations by demon-

\section{References}

1. Bailey B, Bergeron S, Gravel J, Daoust R: Comparison of four pain scales in children with acute abdominal pain in a pediatric emergency department. Ann Emerg Med 50:379-383, 383.e1-2, 2007

2. Bailey B, Daoust R, Doyon-Trottier E, Dauphin-Pierre S, Gravel J: Validation and properties of the verbal numeric scale in children with acute pain. Pain 149:216-221, 2010

3. Beyer J, Aradine C: Content validity of an instrument to measure young children's perceptions of the intensity of their pain. J Pediatr Nurs 1:386-395, 1986

4. Beyer J, Denyes M, Villarruel A: The creation, validation, and continuing development of the oucher: A measure of pain intensity in children. J Pediatr Nurs 7:335-346, 1992

5. Champion GD, Goodenough B, von Baeyer CL, Thomas W: Measurement of pain by self-report, in Finley GA, McGrath PJ (eds): Measurement of Pain in Infants and Children. Seattle, WA, IASP Press, 1998, pp 123-160

6. Cohen J, Cohen P: Applied Multiple Regression/Correlation Analysis for the Behavioral Sciences. Hillsdale, NJ, Erlbaum, 1983

7. Connelly $M$, Neville K: Comparative prospective evaluation of the responsiveness of single-item pediatric painintensity self-report scales and their uniqueness from negative affect in a hospital setting. J Pain 11: $1451-1460,2010$

8. Connelly $M$ : The verbal numeric rating scale in the pediatric emergency department: What do the numbers really mean? Pain 149:167-168, 2010

9. Corzillius M, Fortin P, Stucki G: Responsiveness and sensitivity to change of SLE disease activity measures. Lupus 8: 655-659, 1999

10. Dworkin RH, Turk DC, Farrar JT, Haythornthwaite JA, Jensen MP, Katz NP, Kerns RD, Stucki G, Allen RR, Bellamy N, Carr DB, Chandler J, Cowan P, Dionne R, Galer BS, Hertz $S$, Jadad AR, Kramer LD, Manning DC Martin S, McCormick CG, McDermott MP, McGrath P. strating that the NRS is a valid measure for pain intensity and unpleasantness, and it is sensitive to change in the weeks following major surgery. Using the NRS to measure both the sensory and affective dimension of children's pain experience has the advantage of facilitating comparisons across these dimensions and reducing the burden on children who need to familiarize themselves with only 1 measurement tool. Future avenues for research include the evaluation of different anchors to measure the pain experience ${ }^{24}$ as well as the examination of how children's familiarity with the NRS influences their self-reported pain experience. In addition, research remains to be done in order to translate self-report pain scores on the NRS into a clinically meaningful tool to guide treatment intervention. Lastly, most studies evaluating the validity of the NRS have examined this scale against other pain scales, which in turn have been validated against other pain scales. ${ }^{8}$ As such, it would be helpful for future research to evaluate the validity of the NRS against other types of pain measurements, including biological markers, qualitative descriptions, and behavioral observations.

Quessy S, Rappaport BA, Robbins W, Robinson JP, Rothman M, Royal MA, Simon L, Stauffer JW, Stein W, Tollett J, Wernicke J, Witter J: Core outcome measures for chronic pain clinical trials: IMMPACT recommendations. Pain 113:9-19, 2005

11. Faul F, Erdfelder E, Buchner A, Lang A-G: Statistical power analysis using $G^{*}$ Power 3.1: Tests for correlation and regression analyses. Behav Res Methods 41:1149-1160, 2009

12. Flaherty SA: Pain measurement tools for clinical practice and research. AANA J 64:133-140, 1996

13. Gidron Y, McGrath PJ, Goodday R: The physical and psychosocial predictors of adolescents' recovery from oral surgery. J Behav Med 18:385-399, 1995

14. Goodenough B, Thomas W, Champion GD, Perrott D, Taplin JE, von Baeyer CL, Ziegler JB: Unravelling age effects and sex differences in needle pain: Ratings of sensory intensity and unpleasantness of venipuncture pain by children and their parents. Pain 80:179-190, 1999

15. Goodenough B, Piira T, von Baeyer CL, Chua K, Wu E, Trieu JDH, Champion GD: Comparing six self-report measures of pain intensity in children. The Suffering Child 8: $1-25,2005$

16. Hester N, Foster R, Kristensen K: Measurement of pain in children: Generalizability and validity of the pain ladder and Pieces of Hurt tool. Adv Pain Res Ther 15:79-84, 1990

17. Hicks $C$, Von Baeyer $C L$, Spafford $P$, van Korlaar I, Goodenough B: The faces pain scale-revisited: Toward a common metric in pediatric pain measurement. Pain 93: 173-183, 2001

18. Jackson SL (ed): Correlational methods and statistics, in Research Methods and Statistics: A Critical Thinking Approach. Belmont, CA, Wadsworth, 2009, 140-162

19. Kashikar-Zuck S, Vaught $M H$, Goldschneider $K R$, Graham TB, Miller JC: Depression, coping, and functional disability in juvenile fibromyalgia syndrome. J Pain 3: 412-419, 2002 
20. Kendrick DB, Strout TD: The minimum clinically significant difference in patient-assigned numeric scores for pain. Am J Emerg Med 23:828-832, 2005

21. Lynch AM, Kashikar-Zuck S, Goldschneider KR, Jones BA: Psychosocial risks for disability in children with chronic back pain. J Pain 7:244-251, 2006

22. McGrath PA, de Veber L, Heam M: Multidimensional pain assessment in children, in Advances in Pain Research and Therapy. Seattle, WA, Proceedings from the 4th world congress on pain, 1985

23. McGrath PA: Pain in Children: Nature, Assessment, and Treatment. New York, NY, Guilford, 1990

24. McGrath PA, Seifert CE, Speechley KN, Booth JC, Stitt L, Gibson MC: A new analogue scale for assessing children's pain: An initial validation study. Pain 64:435-443, 1996

25. McGrath PJ, Walco GA, Turk DC, Dworkin RH, Brown MT, Davidson K, Eccleston C, Finley GA, Goldschneider K, Haverkos L, Hertz SH, Ljungman G, Palermo T, Rappaport BA, Rhodes T, Schechter N, Scott J, Sethna N, Svensson $O K$ Stinson J, von Baeyer $C L$, Walker $L$, Weisman S, White RE, Zajicek A, Zeltzer L: Core outcome domains and measures for pediatric acute and chronic/recurrent pain clinical trials: PedIMMPACT recommendations. J Pain 9:771-783, 2008

26. Miro J, Castarlenas E, Huguet A: Evidence for the use of a numerical rating scale to assess the intensity of pediatric pain. Eur J Pain 13:1089-1095, 2009

27. Palermo TM: Impact of recurrent and chronic pain on child and family daily functioning: A critical review of the literature. J Dev Behav Pediatr 21:58-69, 2000

28. Perrott DA, Goodenough B, Champion GD: Children's ratings of the intensity and unpleasantness of postoperative pain using facial expression scales. Eur J Pain 8: 119-127, 2004

29. Powell CV, Kelly AM, Williams A: Determining the minimum clinically significant difference in visual analog pain score for children. Ann Emerg Med 37:28-31, 2001
30. Reid GJ, McGrath PJ, Lang BA: Parent-child interactions among children with juvenile fibromyalgia, arthritis and healthy controls. Pain 113:201-210, 2005

31. Salaffi $F$, Stancati A, Silvestri CA, Ciapetti A, Grassi W: Minimal clinically important changes in chronic musculoskeletal pain intensity measured on a numerical rating scale. Eur J Pain 8:283-291, 2004

32. Sarlani E, Farooq N, Greenspan JD: Gender and laterality differences in thermosensation throughout the perceptible range. Pain 106:9-18, 2003

33. Shields BJ, Palermo TM, Powers JD, Grewe SD, Smith GA: Predictors of a child's ability to use a visual analogue scale. Child Care Health Dev 29:281-290, 2003

34. Stinson JN, Kavanagh T, Yamada J, Gill N, Stevens B: Systematic review of the psychometric properties, interpretability and feasibility of self-report pain intensity measures for use in clinical trials in children and adolescents. Pain 125:143-157, 2006

35. Voepel-Lewis T, Burke CN, Jeffreys N, Malviya S, Tait AR: Do 0-10 Numerical Rating scores translate into clinically meaningful pain measures for children? Anesth Analg 112: 415-421, 2011

36. von Baeyer CL: Children's self-reports of pain intensity: Scale selection, limitations and interpretation. Pain Res Manag 11:157-162, 2006

37. von Baeyer CL, Spagrud L, McCormick JC, Choo E, Neville K, Connelly MA: Three new datasets supporting use of the Numerical Rating Scale (NRS-11) for children's selfreports of pain intensity. Pain 143:223-227, 2009

38. Walker LS, Greene JW: The functional disability inventory: Measuring a neglected dimension of child health status. J Pediatr Psychol 16:39-58, 1991

39. Williamson A, Hoggart B: Pain: A review of three commonly used pain rating scales. J Clin Nurs 14:798-804, 2005

40. Wong D, Baker C: Pain in children: Comparison of assessment scales. Pediatr Nurs 14:9-17, 1988 